\title{
Optimal dispersion precompensation by pulse chirping
}

\author{
Ira Jacobs and John K. Shaw
}

\begin{abstract}
For the procedure of dispersion precompensation in fibers by prechirping, we found that there is a maximum distance over which a pulse initially compressed by prechirping can return to its original width. The distance constraint comes in the form of a mathematical relationship involving the distance, dispersion, initial pulse width, and peak power, implying that the restriction governs all the fiber parameters. Simple closed-form approximations for the constraint and for the corresponding required prechirp are derived on the basis of a variational approach. The validity of the analytical formulas is confirmed by split-step Fourier numerical simulation. (C) 2002 Optical Society of America

OCIS codes: $\quad 060.2330,060.4370,060.4510$.
\end{abstract}

\section{Introduction}

Optical pulse prechirping has recently emerged as a critical design tool in fiber-optic systems, especially with the increased significance of the chirped return to zero format. ${ }^{1-4}$ Prechirping accomplishes various objectives including suppression of four-wave mixing, ${ }^{3,4}$ the lowering of pulse-width fluctuations, ${ }^{5}$ spectral compression, ${ }^{6,7}$ and the generation of transformlimited output pulses. ${ }^{4}$ In dispersion-managed soliton systems a chirped pulse can experience pulse width and phase fluctuations but, with the correct prechirp, can be made to return to its exact initial conditions after a given propagation distance. ${ }^{8}$ Likewise, equal input and output spectral widths can be arranged, at least approximately, for an appropriately prechirped pulse. ${ }^{7}$ Equal input and output pulse and spectral widths are important in the design of single fiber links and periodic dispersion maps because the return to initial widths, with opposite sign of chirp, can simplify the dispersion map.

However, finding the exact initial chirp required for a given output condition is difficult. 5,7 In fact, as we show, for a given propagation distance in a fiber with fixed dispersion, it is not always possible to achieve a desired output pulse width and chirp. Specifically, there is no initial chirp that will cause

I. Jacobs and J. K. Shaw (shaw@math.vt.edu) are with the Bradley Department of Electrical and Computer Engineering, Virginia Polytechnic Institute and State University, Blacksburg, Virginia 24061-0111.

Received 23 April 2001; revised manuscript received 15 October 2001.

0003-6935/02/061057-06\$15.00/0

(C) 2002 Optical Society of America the output and input widths to be equal unless the fiber length, dispersion, input power, and initial width satisfy a particular constraint, which we derive in this paper. Using the variational method, ${ }^{9-11}$ we obtain a simple, closed-form expression for the correct prechirp. We verify all approximation formulas by split-step Fourier numerical simulations and consider both the normal and the anomalous dispersion cases.

In Appendix A we address the corresponding prechirp problem for the time-bandwidth product (TBP) (rather that the temporal pulse width) and discuss some related research in the literature.

\section{Optimal Chirp}

The nonlinear Schrodinger equation can be approximated by the lossless normalized equation

$$
j \psi_{z}=\left(\beta_{2} / 2\right) \psi_{t t}-\gamma P_{0}|\psi|^{2} \psi \quad[\psi(0,0)=1]
$$

when $P_{0}$ is taken to be the average power between amplifiers over a span. ${ }^{9}$ In addition to Eq. (1), we adopt the variational hypothesis ${ }^{10,12}$

$$
\psi(z, t)=A(z) \exp \left\{-t^{2}\left[\frac{1}{2 a(z)^{2}}-j b(z)\right]\right\}
$$

for initially Gaussian solutions of Eq. (1). Coupled equations between the pulse width $a(z)$ and the chirp $b(z)$ can be derived in the form ${ }^{9,10}$

$$
\begin{aligned}
a_{z} & =-2 \beta_{2} a(z) b(z), \\
a b_{z} & =-2 \beta_{2} a(z) b(z)^{2}+\frac{\beta_{2}}{2 a(z)^{3}}+\frac{\alpha_{0} \gamma P_{0}}{2 \sqrt{2} a(z)^{2}},
\end{aligned}
$$


where $a_{0}=a(0)$ and the subscript $z$ denotes a derivative. If the second part of Eqs. (3) is substituted into the derivative of the first part, one obtains the second-order equation ${ }^{9}$

$$
a_{z z}=\frac{\beta_{2}^{2}}{a(z)^{3}}+\frac{\beta_{2} a_{0} \gamma P_{0}}{\sqrt{2} a(z)^{2}}
$$

involving only the width. An input Gaussian to Eq. (1) satisfies $\psi(0, t)=\exp \left[-t^{2}(1+j C) /\left(2 a_{0}^{2}\right)\right]$, where $C$ is the dimensionless chirp parameter ${ }^{12}$; therefore we have the identification $b_{0}=b(0)=-C /\left(2 a_{0}{ }^{2}\right)$. The initial conditions to accompany Eq. (4) are thus $a(0)$ $=a_{0}$ and $a_{z}(0)=C \beta_{2} / a_{0}$. To further simplify $a(z)$, we expand the squared width in a series $a(z)^{2}=c_{0}+$ $c_{1} z+c_{2} z^{2}+\cdots$ and evaluate the constants using Eqs. (3) and (4). To second order, the series is ${ }^{9}$

$$
a(z)^{2} \cong a_{0}{ }^{2}+2 \beta_{2} C z+\left(\frac{\beta_{2}{ }^{2}}{a_{0}{ }^{2}}+\frac{\beta_{2} \gamma P_{0}}{\sqrt{2}}+\frac{{\beta_{2}}^{2} C^{2}}{a_{0}{ }^{2}}\right) z^{2} .
$$

We apply relation (5) to the normal $\left(\beta_{2}>0\right)$ and anomalous $\left(\beta_{2}<0\right)$ dispersion cases separately.

Regarding the postulate of Eq. (2), the Gaussian is the most commonly used pulse shape for analytical studies and is particularly convenient for the variational approach, whose validity is based on the fact that Gaussian input pulses remain Gaussian during propagation in a linear fiber ${ }^{10}$ and nearly Gaussian in a fiber with moderate nonlinearity. Our primary purpose in using the Gaussian postulate is to obtain simple analytical results that give insight into the relationships between the various parameters affecting system performance and that can then be used to obtain appropriate input conditions for more detailed simulations. A shape postulate analogous to Eq. (2) is also available for hyperbolic secant pulses, for which equations corresponding to Eqs. (3)-(5) can be obtained. A variational approach for other initial pulse shapes, such as rectangular or super-Gaussian, is not suitable because these pulses do not maintain their approximate functional form during propagation.

\section{A. Normal Dispersion}

For $\beta_{2}>0$ it follows from Eq. (4) that the width $a(z)$ grows linearly for large $z$, so that $a^{2}(z)$ is asymptotically quadratic. Thus relation (5) is not only valid for small $z$ but is actually the correct asymptotic form for large $z$. This is also true in the anomalous dispersion case, which is discussed in Subsection 2.C, if the nonlinearity parameter $N=\left(a_{0}^{2} \gamma P_{0} /\left|\beta_{2}\right|\right)^{1 / 2}$ is sufficiently small.

It is convenient to write relation (5) in dimensionless form. Noting that the chirp parameter $C$ is already dimensionless, we introduce the variables $\Delta=$ $\beta_{2} z, F=\left(\gamma P_{0} z / \sqrt{2}\right), u=\Delta / a_{0}{ }^{2}$. Then relation (5) can be written as

$$
\frac{a(z)^{2}}{a_{0}^{2}} \cong 1+u^{2}\left(1+C^{2}\right)+u(2 C+F),
$$

where $C$ and $u$ are dimensionless; $|u|$ is the ratio of physical length to dispersion length (defined below).

Consider a fiber with fixed dispersion $\beta_{2}>0$ and length $z=L_{1}$. If $C<0$, then from Eqs. (3) the initial pulse slope is negative, $a_{z}(0)<0$, and the pulse width initially compresses. However, $a(z)$ is eventually increasing, so if the initial width $a_{0}$ and length $L_{1}$ are fixed, it may be that equal input and output widths, $a\left(L_{1}\right)=a_{0}$, can be achieved. Setting the right side of relation (6) equal to 1 at $z=L_{1}$ and dividing by $u^{2}$, we obtain

$$
C^{2}+\frac{2 C}{u}+\left(1+\frac{F}{u}\right)=0 .
$$

Note that $(F / u)=\left(a_{0}^{2} \gamma P_{0} / \beta_{2} \sqrt{2}\right)=\left(L_{D} / L_{N L}\right) / \sqrt{2}=$ $N^{2} / \sqrt{2}$, where $L_{D}$ and $L_{N L}$ are the familiar dispersion and nonlinear lengths given by $L_{D}=\left(a_{0}{ }^{2} / \beta_{2}\right)$ and $L_{N L}=\left(1 / \gamma P_{0}\right)$, and $N^{2}=L_{D} / L_{N L}{ }^{12} \quad$ Because $F / u=N^{2} / \sqrt{2}$, Eq. (7) becomes

$$
C^{2}+\frac{2 C}{u}+\left(1+\frac{N^{2}}{\sqrt{2}}\right)=0,
$$

whose smaller magnitude (plus sign in the quadratic formula) solution is

$$
C=\frac{-1+\left\{1-u^{2}\left[1+\left(N^{2} / \sqrt{2}\right)\right]\right\}^{1 / 2}}{u} .
$$

For $C$ to be real it is necessary that

$$
u^{2}\left(1+\frac{N^{2}}{\sqrt{2}}\right) \leq 1
$$

We chose the quadratic root $C$ with a smaller modulus to minimize the spectral width; see Eq. (11) below. Both roots are negative, consistent with the fact that the chirp parameter and dispersion parameter $\beta_{2}$ must be of opposite sign. According to Eq. (9) and inequality (10), there is a maximum distance over which a chirped pulse can return to its initial width. The maximum normalized distance is $u_{\max }$ $=\left[1+\left(N^{2} / \sqrt{2}\right)\right]^{-1 / 2}$ given by inequality $(10)$, and the required initial chirp $C$ is given by Eq. (9). Inequality (10) can be viewed as restrictive of any or all parameters in $\beta_{2} L_{1}\left[1+\left(N^{2} / \sqrt{2}\right)\right] \leq a_{0}^{2}$. Because $u_{\max } \leq 1$, then the maximum distance at which prechirp can be used is less than the dispersion length. More generally, the maximum distance is bounded by $\min \left[L_{D},\left(\sqrt{2} L_{D} L_{N L}\right)^{1 / 2}\right]$. When $u$ is at the maximum, $C$ has the value $C=-1 / u$. Some representative parameter values will be given to illustrate both the single- and multiple-pulse cases.

To verify that there is an optimum chirp and maximum distance, we ran split-step Fourier simulations for representative values of $N$ and $u$. These are summarized in Fig. 1 in which the required chirp $C$ that guarantees $a\left(L_{1}\right)=a_{0}$ is plotted against the dimensionless length $u$ for values of $N=0,0.2,0.5$, 1.0. The maximum $u$ values are $u_{\max }=1,0.98,0.92$, and 0.77 in Figs. 1(a)-1(d), respectively. The split- 
(a)
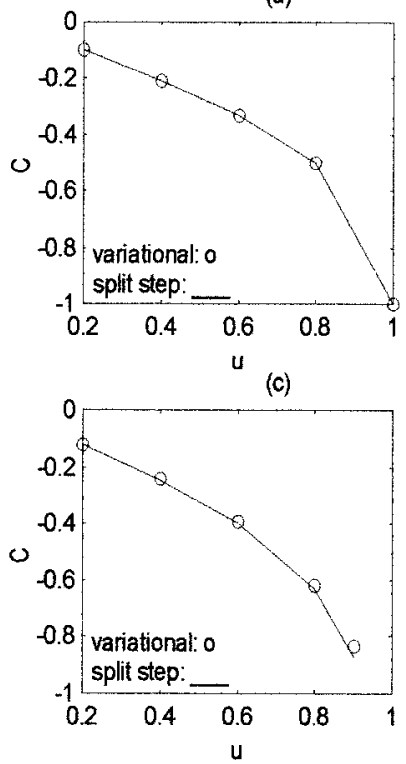

(b)
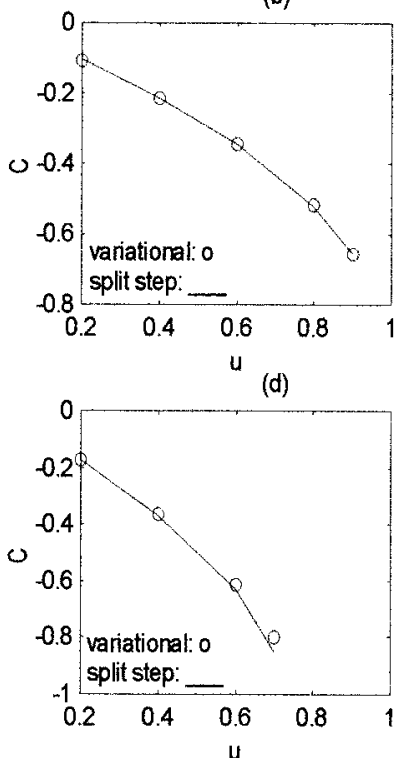

Fig. 1. Comparison of the variational and split-step calculations of the value of $C$ required to ensure equal input and output widths at the dimensionless propagation distance $u$. Parameters are (a) $N=0, u_{\max }=1$; (b) $N=0.2, u_{\max }=0.98$; (c) $N=0.5, u_{\max }=0.92$; (d) $N=1, u_{\max }=0.77$.

step simulation requires a search procedure to find $C$; we simplified the search by using Eq. (9) as the initial estimate. Despite its simplicity, Eq. (9) thus provides an accurate prediction of the required prechirp.

In Fig. 2(a) the ratio of the output-to-input rms pulse widths, as determined from split-step Fourier

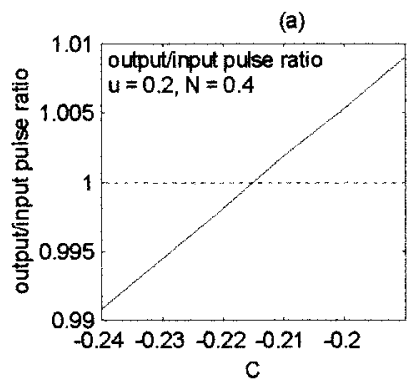

(c)

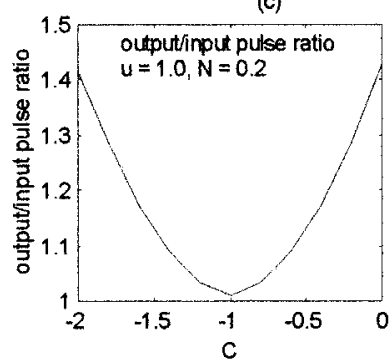

(b)

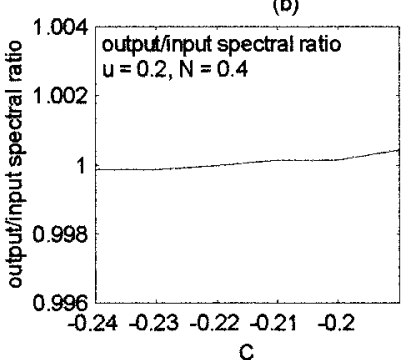

(d)

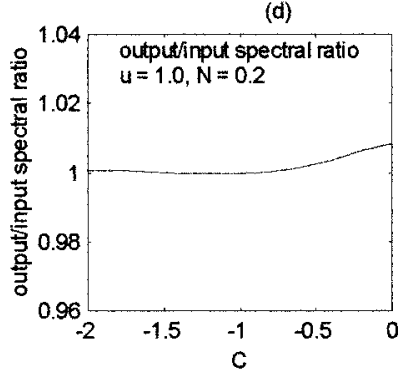

Fig. 2. (a) Ratio of output to input pulse widths for $u=0.2, N=$ $0.4, u_{\max }=0.98$ for various $C$ values near the perfect compensating chirp $C=-0.215$. (b) Ratio of output-to-input spectral widths for the same data as (a). (c) Ratio of output-to-input pulse widths near the minimum $C=-1$ for $N=0.2$ and $u=1>u_{\max }=0.98$. (d) Ratio of output-to-input spectral widths for the same data as (c). All data were obtained by split-step calculation. simulation, is plotted against $C$ for the case of $u=0.2$ and $N=0.4\left(u_{\max }=0.98\right)$. For these parameters, Eq. (9) indicates that the optimum chirp should be $C=-0.215$ [see also Fig. 1(b)]. As can be seen in Fig. 2(a), the ratio of the output-to-input rms pulse widths does indeed occur for $C=-0.215$, in agreement with the analytical result.

In Fig. 2(b) the ratio of the output-to-input rms spectral widths is plotted against $C$, again with the split-step numerical method. Equal input and output spectral widths are obtained at approximately $C=-0.22$. The fact that the same chirp results in restoration of both the initial temporal and the spectral widths can also be shown analytically. The spectral half-width at half-maximum $\Delta \omega(z)$ is given approximately by

$$
\Delta \omega(z)^{2}=\frac{1+C^{2}}{a_{0}^{2}}+\left(\sqrt{2} \gamma P_{0} / \beta_{2}\right)\left(1-\frac{a_{0}}{a(z)}\right) .
$$

[We can derive this formula by taking the Fourier transform of the Gaussian of Eq. (2).] Thus the initial spectral width $\Delta \omega(0)$ equals the output of Eq. (11) exactly when the input and output pulse widths are the same, $a(z)=a_{0}$, so the variational prediction for the spectral crossing is also $C=-0.215$.

\section{B. Case $u>u_{\max }$}

We turn to the case where $u>u_{\max }$ and normal dispersion (similar results hold for anomalous dispersion). For such $u$ the quotient $a(z)^{2} / a_{0}{ }^{2}$ cannot equal 1 , but it can be minimized. For fixed $z=L_{1}$, we set the derivative of the right side of relation (6) to 0 ; solving for $C$ gives $C=-1 / u$. Substituting $C=$ $-1 / u$ into relation (6) gives $\left[a(z)^{2} / a_{0}{ }^{2}\right]=u^{2}+u F>$ $u_{\max }{ }^{2}+u_{\max } F$ because $u>u_{\max }$. However, the relations $u_{\max }{ }^{2}=\left[1+\left(N^{2} / \sqrt{2}\right)\right]^{-1}$ and $\left(N^{2} / \sqrt{2}\right)=$ $(F / u)$ imply that $u_{\max }{ }^{2}+u_{\max } F=1$ and therefore $\left[a(z)^{2} / a_{0}^{2}\right]>1$. The same argument shows that $\left[a(z)^{2} / a_{0}^{2}\right]<1$ at the $C$ giving minimum width when $u<u_{\max }$, and this provides an insight into why there should be two values [the plus and minus in the quadratic formula leading to Eq. (9)] of $C$ that yield $a\left(L_{1}\right)=a_{0}$. In fact, setting $a\left(L_{1}\right)=a_{0}$ means that we are operating at a value above the minimum and so there should be two crossings.

Figure 2(c) shows $C$ plotted against the ratio of the output-to-input widths in the neighborhood of the optimum $C=-1$ for the case $N=0.2$ and $u=1$ ( $u>$ $\left.u_{\max }\right)$. All the widths in Fig. 2(c) were found by splitstep calculations. In Fig. 2(d) we show the ratio of the rms output-to-input spectral widths plotted against $C$ in the same range, with $N=0.2$ and $u=1$, also calculated by the split-step method. The small variation of the spectral ratios reflects the fact that we are operating in a nearly linear regime.

To summarize, there is a maximum $u$ for which the input and output pulse and spectral widths can be made equal. The explicit relationships of Eq. (9), relation (10), and $u_{\max }{ }^{2}=\left[1+\left(\mathrm{N}^{2} / \sqrt{2}\right)\right]^{-1}$ provide simple and accurate means of computing and relating the parameters involved. Note that Eq. (11) shows 
that the spectral width compresses with negative chirp under normal dispersion. This effect has been observed experimentally and in simulations. ${ }^{6}$

\section{Anomalous Dispersion}

The above results carry over partially to the anomalous dispersion case, but the situation is more complicated because, for $\beta_{2}<0$ in Eq. (1), the initial pulse may evolve into a soliton if $N$ is sufficiently large. The width of a pure soliton is either constant or periodic, and in either case the pulse width always returns to, or never varies from, its initial value. A further complication is that a pulse whose initial width and peak power are such that the pulse would asymptotically evolve into a soliton, if it were unchirped, loses this asymptotic property if it is too severely chirped. ${ }^{13}$ Note that we are also considering Gaussian pulses that cannot themselves be pure solitons (which are hyperbolic secants).

For anomalous dispersion, $u$ is replaced by $-u$ in the steps leading to Eq. (9), which formally becomes

$$
\begin{aligned}
C & =\frac{1-\left\{1-u^{2}\left[1-\left(N^{2} / \sqrt{2}\right)\right]\right\}^{1 / 2}}{u}, \\
u^{2} & \leq u_{\max }^{2}=\left(1-\frac{N^{2}}{\sqrt{2}}\right)^{-1},
\end{aligned}
$$

where we chose the smaller of two positive roots so that $C>0$. If $N^{2}<\sqrt{2}$ then we obtain a real valued $u_{\max }$ and Eqs. (12) give the conditions in which equal input and output widths can be achieved, along with the appropriate chirp $C$. Sufficiently large $N$ values imply soliton formation, ${ }^{12}$ and so the series in relation (5) is no longer asymptotically correct. Note that in the anomalous dispersion case $u_{\max }$ increases with $N$, which is opposite to the normal dispersion case. In Figs. 3(a) and 3(b) we plot the required chirp $C$ that guarantees equal input and output widths against the dimensionless length $u$ for $N=0.2$ and $N=0.4$, respectively, in the anomalous dispersion case. Note that $C$ is positive and increases with $u$. As in Fig. 1, the variational prediction [open circles in Figs. 3(a) and 3(b)] is quite close to the actual $C$ value as calculated by the split-step method [solid curves in Figs. 3(a) and 3(b)]. The predicted $u_{\max }$ values for $N=0.2$ and $N=0.4$ are 1.03 and 1.13 , respectively, according to Eq. (12). But split-step analysis reveals these values to be 1.00 and 1.06 , respectively, reflecting the fact that the variational procedure is not exact. In Figs. 3(a) and 3(b) we plotted $u$ values running up to $u=1$, rather than to $u_{\text {max }}$, for simplicity.

\section{Multiple Pulses}

The results Eq. (9), inequality (10), and Eq. (12) carry over to multiple pulses if the nonlinearity level $N$ is not too severe. To illustrate we numerically simulated the propagation of twin, identically chirped Gaussian pulses under normal dispersion and demonstrate the results in Figs. 3(c) and 3(d). We used ${ }^{12}$
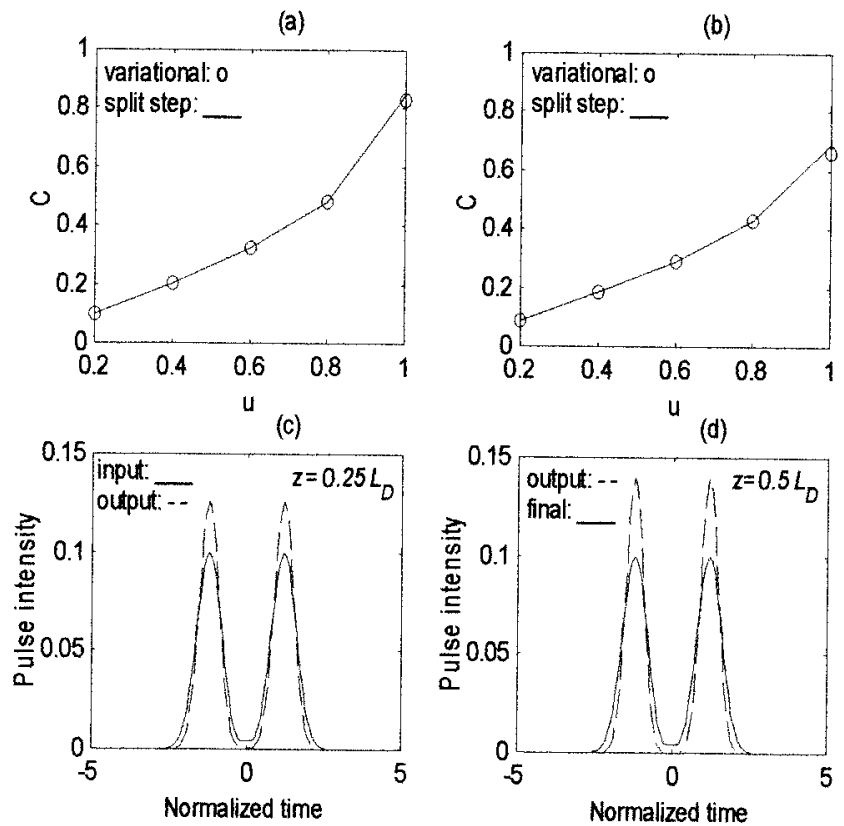

Fig. 3. (a) (b) Comparison of the variational and split-step calculations of the value of $C$ required to assure equal input and output widths at the dimensionless propagation distance $u$ for $0.2 \leq u \leq$ 1 in the anomalous dispersion case. Parameters are (a) $N=0.2$, $u_{\max }=1.03$, (b) $N=0.4, u_{\max }=1.13$. (c) (d) Evolution of twin identically chirped Gaussian pulses under normal dispersion. (c) The dashed curve is the pulse intensity at $z=0.25 L_{D}$ and the solid curve is the input intensity. (d) The dashed curve is the pulse intensity at $z=0.5 L_{D}$ and the solid curve is the output (final) intensity at $z=L_{D}$. The nonlinearity parameter is $N^{2}=0.25$ in both (c) and (d).

$n_{2}=3 \times 10^{-20} \mathrm{~m}^{2} / \mathrm{W}$ and an effective area of 120 $\mu \mathrm{m}^{2}$, giving a nonlinearity constant of $\gamma=0.0101$ $\mathrm{mW}^{-1} \mathrm{~km}^{-1}$. Using a peak power of $P_{0}=0.1 \mathrm{~mW}$, we obtain a nonlinear length of $L_{N L}=\left(1 / \gamma P_{0}\right)=990$ $\mathrm{km}$. In Fig. 3(c) and 3(d) we took $D=-2 \mathrm{ps}(\mathrm{km} /$ $\mathrm{nm})\left(\beta_{2}=2.55 \mathrm{ps}^{2} / \mathrm{km}\right)$ and an input pulse width of 25 ps (bit rate $R_{b}=10$ Gbytes $/ \mathrm{s}$ ). For a single pulse this gives a dispersion length of $L_{D}=245 \mathrm{~km}, N^{2}=$ $0.25, u_{\max }=0.92, z_{\max }=u_{\max } L_{D}=225 \mathrm{~km}$, and $C=$ -1.09 by Eq. (9) and inequality (10) for $u=u_{\max }$. According to Eq. (9) and inequality (10), we expect maximum compression at $z=0.5 L_{D}$ and that the pulse at $z=L_{D}$ should be close to the input pulse. The output pulse intensities at $0.25 L_{D}$ and $0.5 L_{D}$ are shown in Figs. 3(c) and 3(d), respectively, by dashed curves. The input pulse intensity is superimposed as a solid curve in Fig. 3(c), and the output intensity at $z=L_{D}$ is the solid curve in Fig. 3(d); the output is indistinguishable from the input for these parameter values. The plots are with respect to time $\tau=t / a_{0}$ normalized by initial width $a_{0}$, and so the initial pulse has a unit half-width at $z=0$. The initial pulse centers are separated by three half-widths, which allows significant interaction near $\tau=0$ at the input to the fiber. Note that compression resulting from the chirp removes pulse overlap at $z=0.25 L_{D}$. The maximum compression takes place at $z=0.5 L_{D}$. Figures 3(c) and 3(d) show that the phenomenon of 
compression by chirping not only applies to multiple pulses, but that the parameter relationships of Eq. (9) and inequality (10) also carry over. Thus a further advantage of prechirping, when individual pulses are not allowed to expand beyond their initial width, is to ensure that interpulse interaction effects are small.

\section{Summary}

We have found simple, approximate formulas, accurate for the parameter ranges of interest, for the correct chirp in precompensation. We find that it is not always possible to arrange for a chirped pulse, whose width fluctuates during propagation, to return to its initial width in a single section of fiber with the fixed dispersion constant. In the case of normal dispersion, the limiting constraint is contained in the simple formula $\beta_{2} L\left[1+\left(N^{2} / \sqrt{2}\right)\right] \leq a_{0}{ }^{2}$. We have also shown analytically, and confirmed by simulation, that returning the pulse to its initial width at the output of the fiber also returns the spectral width to its initial value. In effect, the chirp parameter at the output is the negative of the value at the input. It would then be possible to have a following section of fiber with the opposite signed dispersion parameter that would again change the sign of the chirp parameter, thereby returning the pulse to the conditions at the input to the first fiber.

We believe that these results are significant in the understanding of the nature of prechirp and the parameter ranges over which precompensation can be used in the design of both single-link systems and periodic dispersion maps.

\section{Appendix A}

\section{Time-Bandwidth Product}

We can also consider the TBP as a design parameter. ${ }^{4}$ Using the calculations leading to Eq. (11), one can show that $\Delta \omega(z)^{2} a(z)^{2}=1+\left[a(z)^{2} a_{z}(z) / \beta_{2}{ }^{2}\right]$. Then the TBP achieves a minimum of 1 when $a_{z}=0$. Because $2 a a_{z}$ is the derivative of $a^{2}(z)$, then we can minimize the TBP relative to $C$ using relation (5) or (6). A simple calculation yields $a(z) a_{z}(z) / \beta_{2}=u(1+$ $\left.C^{2}\right)+(C+F)$. Thus the TBP $\Delta \omega(z) a(z)$ is 1 when

$$
\begin{aligned}
& C=\left(-1+\left\{1-4 u^{2}\left[1+\left(N^{2} / \sqrt{2}\right)\right]\right\}^{1 / 2}\right) / 2 u, \\
& u \leq u_{\max }=1 / 2\left[1+\left(N^{2} / \sqrt{2}\right)\right]^{1 / 2} .
\end{aligned}
$$

In particular, a transform-limited output pulse (TBP equaling 1) can be achieved only under the restriction $2 \beta_{2} z\left[1+\left(N^{2} / \sqrt{2}\right)\right]^{1 / 2} \leq a_{0}^{2}$ and for the chirp parameter $C$ as given above. In Ref. 4 the authors used an approach similar to the variational method, called the moment method, to study the effects of pulses that are transform limited at the output. They note that it is not always possible to achieve transform-limited outputs but do not give an explicit formula such as inequality (10).

\section{Related Literature}

A quadratic series method was used in Ref. 14 to model pulse behavior in fibers with unchirped pulses. Governing equations for the width were based on explicitly computable integral moments. Liao et al. ${ }^{8}$ used a variational technique coupled with two quadratic power series to find the unique prechirp $C$ and peak power $P_{0}$ that induces a pulse propagating under anomalous dispersion to return to its exact initial width and chirp at the amplifier. The authors in Ref. 8 did not consider normal dispersion, and the nonlinearity levels fall in the range $N^{2}>\sqrt{2}$, making our results and Ref. 8 essentially complementary (see our remarks in Subsection 2.C). The two quadratic series in Ref. 8 are expansions in a perturbation parameter $z_{A}$ (amplifier spacing divided by dispersion length) whose coefficients are corrections to the pulse width and chirp. A quadratic series analogous to relation (5) has been used ${ }^{15}$ to calculate the point of maximum rms pulse-width compression in a dispersive, nonlinear segment of a chirped-pulse amplification device; there is no prechirp analysis in Ref. 15.

\section{References}

1. N. Bergano, "Undersea fiberoptic cable systems," Opt. Photon. News 11, 20-25 (2000).

2. N. S. Bergano, C. R. Davidson, M. Ma, A. Pilipetskii, S. G. Evangelides, H. D. Kidorf, J. M. Darcie, E. Goluvchenko, K. Rottwitt, P. C. Corbett, R. Menges, M. A. Mills, B. Pedersen, D. Peckham, A. A. Abramov, and A. M. Vengsarkar, "320 Gb/s WDM transmission $(64 \times 5 \mathrm{~Gb} / \mathrm{s})$ over $7,200 \mathrm{~km}$ using large mode fiber spans and chirped return-to-zero signals," in Optical Fiber Communication Conference (OFC), Vol. 2 of 1998 OSA Technical Digest Series (Optical Society of America, Washington, D.C., 1998), pp. PD12-1-PD12-4.

3. S. Cundiff, B. Collins, L. Boivin, M. Nuss, K. Bergman, W. Knox, and S. Evangelides, "Propagation of highly chirped pulses in fiber-optic communication systems," J. Lightwave Technol. 17, 811-816 (1999).

4. T. Yang, W. Kath, and S. Evangelides, "Optimal prechirping for dispersion-managed transmission of return-to-zero pulses," in Optical Fiber Communication Conference, (OFC), Vol. 5 of 1999 OSA Technical Digest Series (Optical Society of America, Washington, D.C., 1999), pp. 249-251.

5. Y. Wen and X. Yang, "Quasi-transform-limited pulse transmission in dispersion managed soliton system," Photon. Technol. Lett. 11, 433-435 (1999).

6. B. Washburn, J. Buck, and S. Ralph, "Transform-limited spectral compression due to self-phase modulation in fibers," Opt. Lett. 25, 445-447 (2000).

7. M. Zitelli, F. Matera, and M. Settembre, "Single channel transmission in dispersion management links in conditions of very strong pulse broadening: application to $40 \mathrm{~Gb} / \mathrm{s}$ signals on step-index fibers," J. Lightwave Technol. 17, 2498-2505 (1999).

8. Z. Liao, C. McKinstrie, and G. P. Agrawal, "Importance of prechirping in constant-dispersion fiber links with a large amplifier spacing," J. Opt. Soc. Am. B 17, 514-518 (2000).

9. M. Brandt-Pearce, I. Jacobs, J.-H. Lee, and J. K. Shaw, "Optimal input Gaussian pulse width for transmission in dispersive nonlinear fibers," J. Opt. Soc. Am. B 16, 11891196 (1999). 
10. D. Anderson, "Variational approach to nonlinear pulse propagation in fibers," Phys. Rev. A 27, 3135-3145 (1983).

11. V. Grigorian, T. Yu, E. Goluvchenko, C. Menyuk, and A. Pilipetski, "Dispersion-managed soliton dynamics," Opt. Lett. 22, 1609-1611 (1997).

12. G. P. Agrawal, Nonlinear Fiber Optics, 3rd ed. (Academic, New York, 2001).

13. D. Anderson, M. Lisak, and T. Reichel, "Asymptotic propaga- tion properties of pulses in a soliton-based optical-fiber communication system,” J. Opt. Soc. Am. B 5, 207-210 (1988).

14. P. Belanger and C. Pare, "Second-order moment analysis of dispersion-managed solitons," J. Lightwave Technol. 17, 445451 (1999).

15. F. Cattani, D. Anderson, A. Berntson, and M. Lisak, "Effect of self-phase modulation in chirped-pulse-amplification-like schemes," J. Opt. Soc. Am. B 16, 1874-1879 (1999). 